\title{
Effects of hydrogen sulfide on myocardial fibrosis and PI3K/AKT1-regulated autophagy in diabetic rats
}

\author{
TING XIAO, JIAN LUO, ZHIXIONG WU, FANG LI, OU ZENG and JUN YANG \\ Department of Cardiology, The First Affiliated Hospital of University of South China, Hengyang, Hunan 421001, P.R. China
}

Received April 6, 2015; Accepted November 6, 2015

DOI: $10.3892 / \mathrm{mmr} .2015 .4689$

\begin{abstract}
Myocardial fibrosis is the predominant pathological characteristic of diabetic myocardial damage. Previous studies have indicated that hydrogen sulfide $\left(\mathrm{H}_{2} \mathrm{~S}\right)$ has beneficial effects in the treatment of various cardiovascular diseases. However, there is little research investigating the effect of $\mathrm{H}_{2} \mathrm{~S}$ on myocardial fibrosis in diabetes. The present study aimed to investigate the effects of $\mathrm{H}_{2} \mathrm{~S}$ on the progression of myocardial fibrosis induced by diabetes. Diabetes was induced in rats by intraperitoneal injection of streptozotocin. Sodium hydrosulfide (NaHS) was used as an exogenous donor of $\mathrm{H}_{2} \mathrm{~S}$. After 8 weeks, expression levels of cystathionine- $\gamma$-lyase were determined by western blot analysis and morphological changes in the myocardium were assessed by hematoxylin and eosin staining and Masson staining. The hydroxyproline content and fibrosis markers were determined by a basic hydrolysis method and western blot analysis, respectively. Autophagosomes were observed under transmission electron microscopy. Expression levels of autophagy-associated proteins and their upstream signaling molecules were also evaluated by western blotting. The results of the current study indicated that diabetes induced marked myocardial fibrosis, enhanced myocardial autophagy and suppressed the phosphatidylinositol-4,5-bisphosphate 3 -kinase/R AC- $\alpha$ serine/threonine-protein kinase (PI3K/AKT1) signaling pathway. By contrast, following treatment with NaHS, myocardial fibrosis was ameliorated, myocardial autophagy was decreased and the PI3K/AKT1 pathway suppression was reversed. The results of the present study demonstrated that the protective effect of $\mathrm{H}_{2} \mathrm{~S}$ against
\end{abstract}

Correspondence to: Professor Jun Yang, Department of Cardiology, The First Affiliated Hospital of University of South China, 69 Chuanshan Road, Shigu, Hengyang, Hunan 421001, P.R. China E-mail: yangjunincn@163.com

Abbreviations: $\mathrm{H}_{2} \mathrm{~S}$, hydrogen sulfide; NaHS, sodium hydrosulfide; DCM, diabetic cardiomyopathy; ECM, extracellular matrix; CSE, cystathionine- $\gamma$-lyase; STZ, streptozotocin; MMPs, matrix metalloproteinases

Key words: hydrogen sulfide, diabetes, myocardial fibrosis, autophagy, phosphatidylinositol-4,5-bisphosphate 3-kinase/RAC- $\alpha$ serine/threonine-protein kinase diabetes-induced myocardial fibrosis may be associated with the attenuation of autophagy via the upregulation of the PI3K/AKT1 signaling pathway.

\section{Introduction}

Diabetic cardiomyopathy (DCM) is characterized clinically by diastolic dysfunction in the early stage, evolving to systolic dysfunction in the final stage. Impaired ejection fraction is a severe complication that affects a notable proportion of diabetic patients (1). With the rising incidence of diabetes, DCM is an increasingly important disease in the cardiovascular field. Myocardial fibrosis results from a disproportionate increase in collagen deposition in the extracellular matrix (ECM). It is one of the predominant pathological features of DCM (2) and it is a critical determinant of the development of diastolic and systolic dysfunction in patients with DCM (3). However, there are no effective therapeutic strategies currently available that prevent the progression of diabetic myocardial fibrosis. Further elucidation of the mechanisms underlying myocardial fibrosis in diabetic patients is required and potential therapeutic strategies to limit myocardial remodeling in DCM remain of interest to reduce the risk of progression to heart failure.

Hydrogen sulfide $\left(\mathrm{H}_{2} \mathrm{~S}\right)$, which was previously considered to be a highly toxic gas, has been identified as a novel gaseous signaling molecule (4), and similar to carbon monoxide and nitric oxide, it exerts diverse effects on multiple physiological and pathological processes in various tissues and organs (5). Recently, research into $\mathrm{H}_{2} \mathrm{~S}$ in the pathogenesis of cardiovascular disorders has rapidly increased as the roles of $\mathrm{H}_{2} \mathrm{~S}$ in the cardiovascular system, including vasorelaxation, inhibition of vascular remodeling, anti-atherosclerosis and cardioprotection, have been demonstrated (5-8). In the heart, endogenous $\mathrm{H}_{2} \mathrm{~S}$ is predominantly generated from L-cysteine by cystathionine- $\gamma$-lyase (CSE) $(9,10)$. Previous studies have demonstrated that $\mathrm{H}_{2} \mathrm{~S}$ ameliorates myocardial ischemia-reperfusion injury by attenuating cardiomyocyte apoptosis, preserving mitochondrial function $(11,12)$ and alleviating structural and functional deterioration of the left ventricle in ischemia-induced heart failure by attenuating oxidative stress (13). Furthermore, a recent study indicated that the decreased generation of endogenous $\mathrm{H}_{2} \mathrm{~S}$ is important in the development of diabetic nephropathy in diabetic rats and administration of $\mathrm{H}_{2} \mathrm{~S}$ may delay the progress of diabetic nephropathy by reducing mesangial cell proliferation, 
decreasing inflammation and oxidative stress, and inhibiting the activity of the renin-angiotensin system (14). In addition, it has been demonstrated that $\mathrm{H}_{2} \mathrm{~S}$ improves left ventricular function in smoking rats by reducing autophagy (15). However, the effect of $\mathrm{H}_{2} \mathrm{~S}$ on diabetic myocardial fibrosis and its underlying mechanisms remain to be elucidated.

Autophagy is a lysosome-dependent process, which degrades dysfunctional organelles and damaged proteins to maintain cellular homeostasis and structural integrity of the cell. Autophagy represents an evolutionarily conserved process for bulk degradation and recycling of cytoplasmic components (16-18). However, prolonged activation of autophagy may be observed in the heart under several cardiotoxic stressors, including hyperglycemia, ischemia-reperfusion and chronic high pressure load, this accelerates cell death and results in the occurrence of associated cardiovascular disease $(19,20)$.

In the present study, a streptozotocin (STZ)-induced diabetic rat model was used to evaluate the effects of $\mathrm{H}_{2} \mathrm{~S}$ on diabetic myocardial fibrosis, and furthermore, to investigate the effects of $\mathrm{H}_{2}$ S on phosphatidylinositol-4,5-bisphosphate 3-kinase/RAC- $\alpha$ serine/threonine-protein kinase (PI3K/AKT1)-regulated autophagy in order to elucidate its underlying mechanisms.

\section{Materials and methods}

Animals and reagents. The experimental protocol was approved by the Animal Ethics Committee of the University of South China (Hengyang, China). Adult male Sprague-Dawley rats (weight, 288-300 g) were obtained from the SJA Lab Animal Center of Changsha (Changsha, China). The rats were housed in separate cages and had free access to food and water. Animals were kept in a climate-controlled room with a 12-h light/dark cycle. Sodium hydrosulfide (NaHS) was purchased from Sigma-Aldrich (St. Louis, MO, USA). STZ was purchased from MP Biomedicals, LLC (Santa Ana, CA, USA). A hydroxyproline detection kit was obtained from Nanjing Jiancheng Bioengineering Institute (Nanjing, China). Rabbit polyclonal antibody against CSE (cat. no. sc-135203; used at 1:1,000 dilution) was purchased from Santa Cruz Biotechnology, Inc. (Dallas, TX, USA). Rabbit polyclonal anti-collagen I (cat. no. BA0235), rabbit polyclonal anti-collagen III (cat. no. BA0326), rabbit polyclonal anti-matrix metalloproteinase (MMP)7 (cat. no. BA2110), rabbit polyclonal anti-MMP8 (cat. no. BA2201), rabbit polyclonal anti-MMP14 (cat. no. BA1278), rabbit polyclonal anti-tissue inhibitor of metalloproteinase 1 (TIMP1; cat. no. BA3727), rabbit polyclonal anti-transforming growth factor $\beta 1$ (TGF $\beta 1$; cat. no. BA0290), rabbit polyclonal anti-PI3K (cat. no. BA0351), rabbit polyclonal anti-AKT1 (cat. no. BA0631) and rabbit polyclonal anti-glyceraldehyde 3-phosphate dehydrogenase (GAPDH; cat. no. PB0141) were all purchased from Wuhan Boster Biological Technology, Ltd. (Wuhan, China). The dilution rate of these antibodies was 1:400. Furthermore, rabbit monoclonal anti-Beclin1 (cat. no. 3495), rabbit monoclonal anti-autophagy-related protein (Atg; cat. no. 3415), rabbit monoclonal anti-Atg5 (cat. no. 12994) and rabbit monoclonal anti-Atg16 (cat. no. 8089) were purchased from Cell Signaling Technology, Inc, (Danvers, MA, USA). The dilution rate of these antibodies was 1:1,000. Anti-rabbit secondary antibody (cat. no. 074-1506) was obtained from KPL, Inc. (Gaithersburg, MD, USA), which was used at a dilution of 1:8,000. Cell lysis buffer for western blotting, bicinchoninic acid (BCA) protein assay kit and SDS-PAGE gel preparation kit were purchased from Beyotime Institute of Biotechnology (Haimen, China).

Diabetes model. The rats were allowed to acclimatize for 7 days prior to the experiment. Diabetes was induced by a single intraperitoneal injection of STZ $(40 \mathrm{mg} / \mathrm{kg})$ dissolved in $0.1 \mathrm{M}$ sodium citrate buffer ( $\mathrm{pH} 4.4$; prepared from citric acid and trisodium citrate; Sinopharm Chemical Reagent Co., Ltd., Shanghai, China) overnight. In place of sterile water, 5\% glucose solution was administered to STZ-treated rats $24 \mathrm{~h}$ following injection in order to prevent death due to hypoglycemic shock. Following STZ injection (72 h), blood samples were collected from the tail vein to measure blood glucose levels using a blood glucose meter (Sinocare Inc., Changsha, China). Only those rats with blood glucose levels $\geq 16.8 \mathrm{mM}$ were considered to be successful models of diabetes and were recruited into the study (5). The rats with lower glucose levels were subjected to another injection of STZ $(20 \mathrm{mg} / \mathrm{kg})$ until their blood glucose levels were $\geq 16.8 \mathrm{mM}$

Experimental protocol. The experimental rats were divided into four groups as follows ( $n=10$ per group): i) Control group (normal rats); ii) diabetes model (DM) group (diabetes rats); iii) DM + NaHS group (diabetes rats treated with NaHS); and iv) NaHS group (normal rats treated with NaHS). The control and DM groups were intraperitoneally injected with physiological saline for 8 weeks and the DM + NaHS and NaHS groups were intraperitoneally administered with an equivalent volume of NaHS at a dose of $100 \mu \mathrm{mol} / \mathrm{kg}$ for 8 weeks. At the end of the experiment, rats were sacrificed by anesthesia with an intraperitoneal injection of chloral hydrate ( $350 \mathrm{mg} / \mathrm{kg}$; Pharmacy of the Affiliated South China Hospital of the University of South China, Hengyang, China). Hearts were lavaged with ice-cold normal saline and removed.

Histopathological examination. Myocardium samples from the experimental rats were fixed using $4 \%$ paraformaldehyde (Sinopharm Chemical Reagent Co.), dehydrated with alcohol, embedded in paraffin (Sinopharm Chemical Reagent Co.) and cut into 5- $\mu \mathrm{m}$ sections. The sections were stained using a hematoxylin and eosin (H\&E) staining kit (Beyotime Institute of Biotechnology) or a Masson staining kit (Nanjing Senbeijia Biological Technology Co., Ltd., Nanjing, China), and observed under light microscopy (Motic BA210; Motic Medical Diagnostic Systems Co., Ltd., Xiamen, China) at a magnification of $\mathrm{x} 200$.

Hydroxyproline content assay. Myocardial hydroxyproline content was measured by a basic hydrolysis method with a hydroxyproline detection kit according to the manufacturer's instructions. The left ventricular tissue of the rats was cut into $10-\mathrm{mg}$ pieces and added to $1 \mathrm{ml}$ basic hydrolysates. The samples were hydrolyzed at $100^{\circ} \mathrm{C}$ for $20 \mathrm{~min}$. Absorbance was measured at a wavelength of $490 \mathrm{~nm}$ using an automatic enzyme mark reading meter (cat. no. 500; Bio-Rad Laboratories, Inc., Hercules, CA, USA) and the hydroxyproline content was calculated. Results were expressed as micrograms of hydroxyproline per milligram of cardiac tissue (wet weight). 
Transmission electron microscopy analysis. The left ventricular tissue from the harvested hearts in each group was cut into small pieces on ice and fixed in $2.5 \%$ glutaraldehyde (Sinopharm Chemical Reagent Co.), post-fixed in 1\% osmium tetroxide (Absin Bioscience Inc., Shanghai, China) and dehydrated in a series of graded ethanol solutions. Ultrathin sections were cut and stained with uranyl acetate (Shanghai Fortune Biological Technology Co., Ltd., Shanghai, China) and lead citrate (Tanyun Industry Fine Chemical Co., Ltd., Yingkou, China). Samples were observed and images were captured by transmission electron microscopy.

Western blot analysis. Total proteins were extracted in ice-cold radioimmunoprecipitation assay buffer containing protease inhibitors (Beyotime Institute of Biotechnology), and quantified using a BCA protein assay kit. The proteins were denatured, separated by $10 \%$ SDS-PAGE electrophoresis and transferred to a polyvinylidene fluoride membrane (Millipore, Billerica, MA, USA). The membranes were blocked with 5\% skimmed milk in Tris-buffered saline (Tris supplied by Biosharp Co., Hefei, China and $\mathrm{NaCl}$ from Sinopharm Chemical Reagent Co.) with Tween 20 (Wellbiology Co., Ltd. Changsha, China) (TBST) prior to overnight incubation at $4{ }^{\circ} \mathrm{C}$ with antibodies against CSE (1:1,000), collagen I (1:400), collagen III (1:400), MMP7 (1:400), MMP8 (1:400), MMP14 (1:400), TIMP1 (1:400), TGF $\beta 1$ (1:400), PI3K (1:400), AKT1 (1:400), Beclin-1 (1:1,000), Atg3 (1:1,000), Atg5 (1:1,000) and Atg16 (1:1,000). Following washing three times with TBST, the membranes were incubated with horseradish peroxidase-conjugated secondary antibody $(1: 8,000)$ for $1 \mathrm{~h}$ at room temperature. Bands were visualized using an enhanced chemiluminescence detection reagent (Beyotime Institute of Biotechnology) and analyzed with a Molecular Imager VersaDoc MP 5000 system (Bio-Rad Laboratories, Inc., Hercules, CA, USA).

Statistical analysis. Data are expressed as the mean \pm standard deviation. Statistical differences among the groups were assessed by one-way analysis of variance with SPSS software, version 18.0 (SPSS, Inc., Chicago, IL, USA). P<0.05 was considered to indicate a statistically significant difference.

\section{Results}

Expression levels of CSE are decreased in diabetic rat myocardium. To investigate whether diabetes-induced myocardial damage was associated with decreased generation of endogenous $\mathrm{H}_{2} \mathrm{~S}$, the expression level of CSE was measured. As shown in Fig. 1, compared with the control group, there was a significant reduction in CSE in the DM group.

Effect of $\mathrm{H}_{2} \mathrm{~S}$ on diabetes-induced changes in myocardial morphology. In order to evaluate the morphological changes in the myocardium, $\mathrm{H} \& \mathrm{E}$ and Masson staining were conducted at the end of the experiment. Fig. 2 shows the morphological changes observed in the myocardium of the different groups. The H\&E staining (Fig. 2A) demonstrated that in the control group, the myocardial cells were orderly and compactly arranged, and less extracellular matrix was observed. In the DM group, the myocyte cross-sectional area

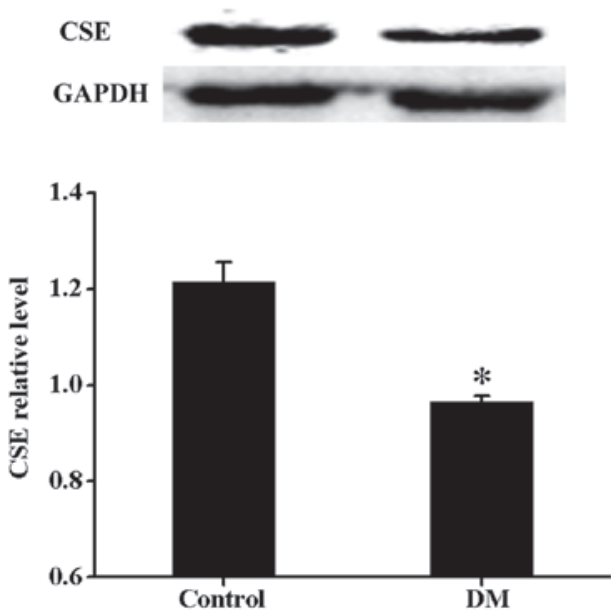

Figure 1. Expression levels of CSE in diabetic rat myocardium samples. Data are expressed as the mean \pm standard deviation $(n=3)$. ${ }^{*} \mathrm{P}<0.05$ vs. the control group. CSE, cystathionine $\gamma$-lyase; DM, diabetes model.

was increased, and relatively disorganized myocardial cells and increased interstitial ECM were observed. However, these above-mentioned changes were markedly reversed in the DM + NaHS group. The results from Masson staining demonstrated the deposition of collagen fibers (Fig. 2B). Blue staining indicated the intensity of fibrosis in the cardiac tissue. There was little evident cardiac fibrosis in the control group, however, increased fibrosis was observed in DM group. Following NaHS treatment, the myocardial fibrosis was markedly alleviated compared with the DM group. H\&E and Masson staining demonstrated that, compared with the control group, there were no notable histological changes in the NaHS group.

Effect of $\mathrm{H}_{2} \mathrm{~S}$ on diabetes-induced change in the hydroxyproline content. As a characteristic of collagen production, hydroxyproline content was determined in the present study (Fig. 3). The myocardial hydroxyproline content in the DM group was significantly higher than that in control group, and it was markedly decreased in the DM + NaHS group compared with the DM group. No significant difference was observed in myocardial hydroxyproline content between the control group and NaHS group.

Effect of $\mathrm{H}_{2} \mathrm{~S}$ on diabetes-induced changes in protein expression levels of collagen I and collagen III. Collagen I and collagen III are the predominant collagen types in the heart, and the effects of $\mathrm{H}_{2} \mathrm{~S}$ on the protein expression levels of collagen I and collagen III were investigated using western blotting (Fig. 4). It was observed that the expression levels of collagen I and collagen III were increased in the myocardium of the DM group compared with those in the control group, while they were reduced in the DM + NaHS group. No significant differences in the expression of collagen I and collagen III were examined between control group and the NaHS group.

Effect of $\mathrm{H}_{2} \mathrm{~S}$ on diabetes-induced changes in the expression levels of MMP7, MMP8, MMP14 and TIMP1. The balance of 
A

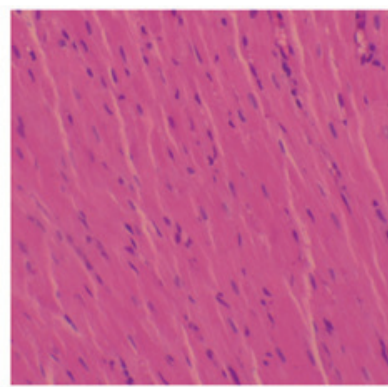

B

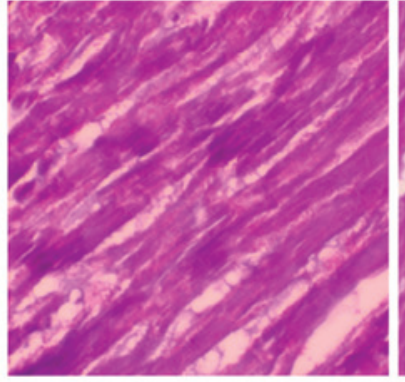

Control
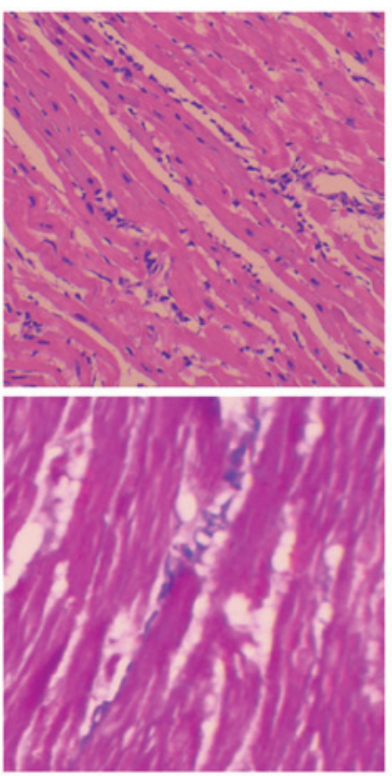

DM
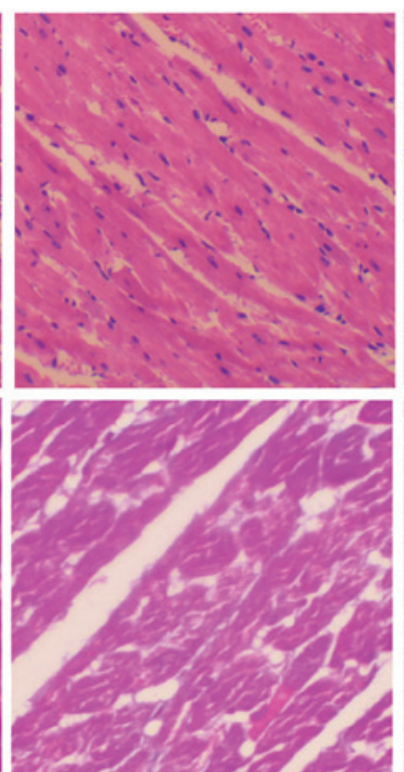

DM+NaHS
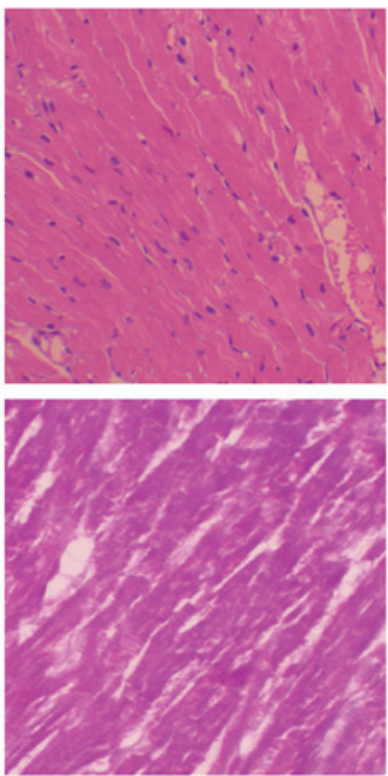

NaHS

Figure 2. Morphological changes in myocardium samples assessed by (A) hematoxylin and eosin staining and (B) Masson staining. Magnification, x200. DM, diabetes model; NaHS, sodium hydrosulfide.

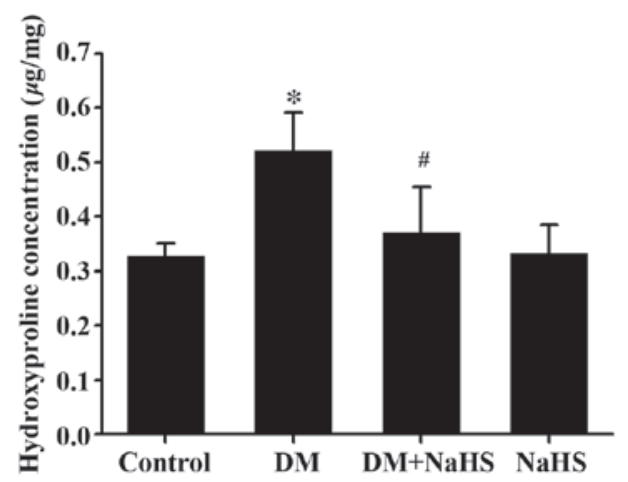

Figure 3. Quantification of myocardial hydroxyproline contents in rats from each group. Data are expressed as the mean \pm standard deviation $(n=3)$. ${ }^{*} \mathrm{P}<0.05$ vs. the control group; ${ }^{\text {"P}}<0.05$ vs. the $\mathrm{DM}$ group. $\mathrm{DM}$, diabetes model; NaHS, sodium hydrosulfide.

MMPs and TIMPs determines the ratio of collagen synthesis and degradation, thus, as it somewhat indicates the status of fibrosis, the expression levels of MMP7, MMP8, MMP14 and TIMP1 were determined (Fig. 5). Results of the western blot analysis indicate that diabetes induced a significant increase in expression levels of MMP7, MMP8 and MMP14, but a decrease in expression levels of TIMP1, and that these changes were markedly reversed by NaHS treatment. No significant difference was observed in the expression of MMP7, MMP8, MMP14 and TIMP1 between the control and NaHS groups.

Effect of $\mathrm{H}_{2} \mathrm{~S}$ on diabetes-induced changes in the expression of TGF $\beta 1$. It is widely accepted that TGF $\beta 1$ is closely associated with fibrogenesis. In the present study, the expression levels of TGF $\beta 1$ were determined by western blot analysis (Fig. 6). Results indicated that the expression levels of TGF $\beta 1$ were markedly increased in the DM group compared with the control group. However, a significant decrease in TGF $\beta 1$ expression was observed in the DM + NaHS group. No significant difference was observed in the expression of TGF $\beta 1$ between the control and NaHS groups.

Effect of $\mathrm{H}_{2} \mathrm{~S}$ on diabetes-induced change in the formation of autophagosomes. To investigate the internal mechanism underlying the beneficial effects of $\mathrm{H}_{2} \mathrm{~S}$ against cardiomyopathy, transmission electron microscopy was adopted to observe autophagosomes (Fig. 7). As demonstrated in Fig. 7, in the DM group, autophagosomes were identified in the myocardium, but not in the DM + NaHS group. In addition, no autophagosomes were observed in the control or NaHS groups.

Effect of $\mathrm{H}_{2} \mathrm{~S}$ on diabetes-induced changes in the expression of autophagy protein markers. In order to further investigate the above results, the expression levels of certain autophagy markers were determined using western blotting (Fig. 8). Compared with the control group, the expression levels of Beclin-1, Atg3, Atg5 and Atg16 were significantly upregulated in the DM group. These autophagy markers were significantly reduced in the DM + NaHS group compared with the DM group. No significant difference was observed in the expression levels of these autophagy markers between the control and NaHS groups.

Effects of $\mathrm{H}_{2} \mathrm{~S}$ on diabetes-induced change in PI3K/AKTI signaling. To further investigate the potential signaling pathways involved in diabetes and/or $\mathrm{H}_{2} \mathrm{~S}$-induced cardiac autophagic response, the expression levels of PI3K and its downstream signaling molecule, AKT1 were determined (Fig. 9). Results from the western blot analysis demonstrated that the expression of PI3K and AKT1 were significantly lower in the DM group than in the control group, however, a significant increase in the expression level of PI3K and AKT1 were observed in the DM + NaHS group. No significant difference 

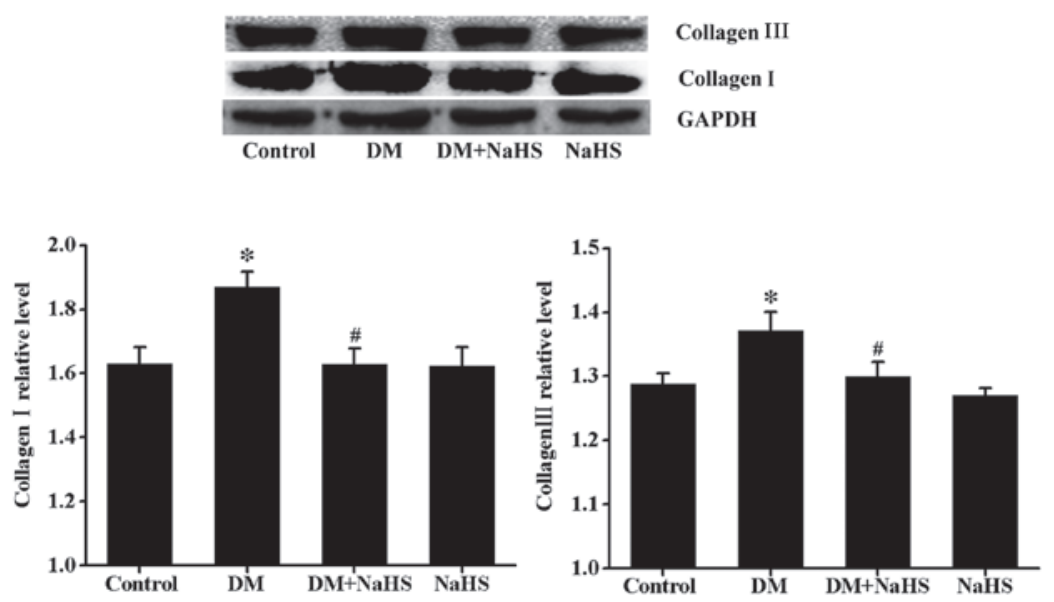

Figure 4. Expression of collagen I and collagen III in the myocardial tissue samples from each group. Data are expressed as the mean \pm standard deviation (n=3). ${ }^{\text {P }}<0.05$ vs. the control group; ${ }^{*} \mathrm{P}<0.05$ vs. the DM group. DM, diabetes model; NaHS, sodium hydrosulfide.
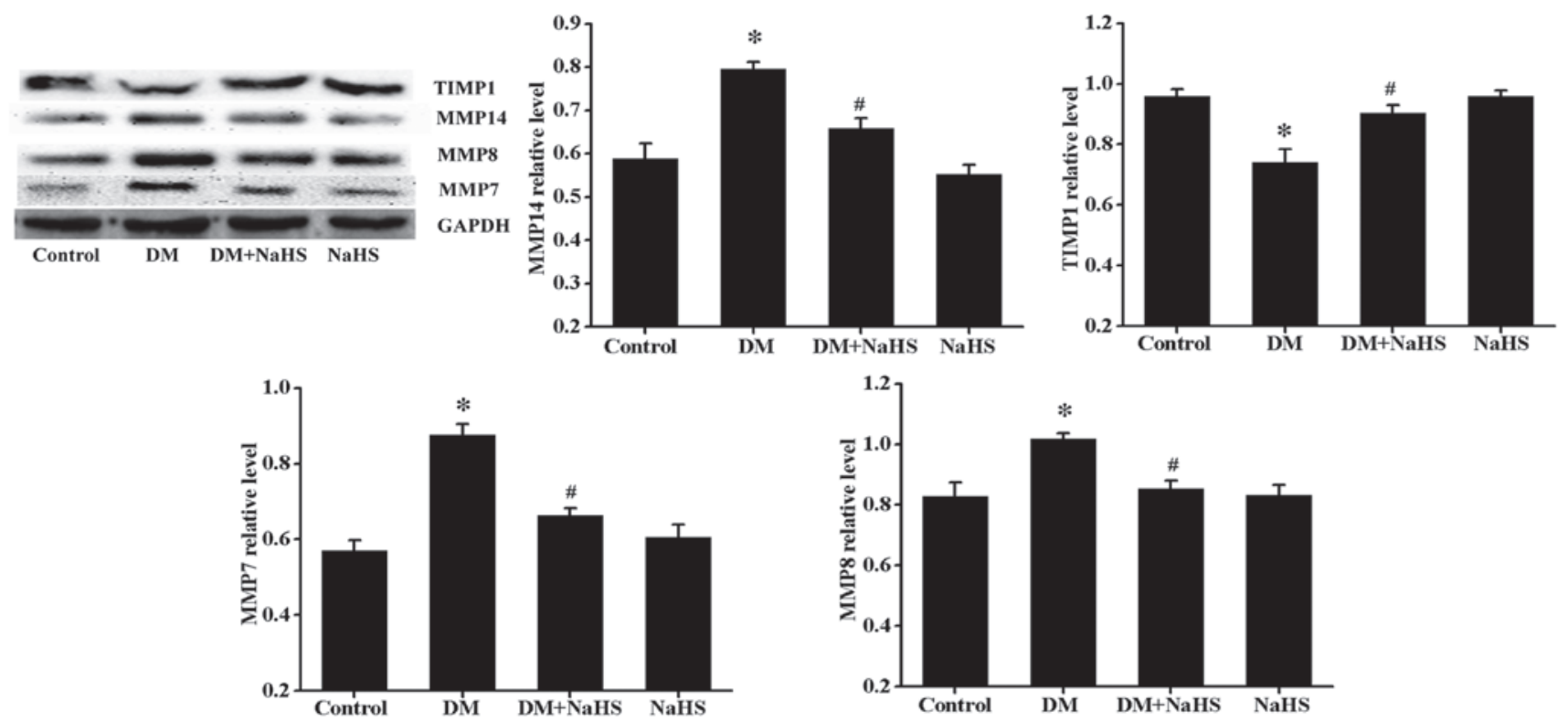

Figure 5. Expression of MMPs and TIMP1 in the myocardial tissues from each group. Data are expressed as the mean \pm standard deviation $(\mathrm{n}=3)$. ${ }^{*} \mathrm{P}<0.05 \mathrm{vs}$. the control group; ${ }^{*} \mathrm{P}<0.05$ vs. the DM group. DM, diabetes model; NaHS, sodium hydrosulfide; MMP, matrix metalloproteinase; TIMP1, tissue inhibitor of metalloproteinases 1.
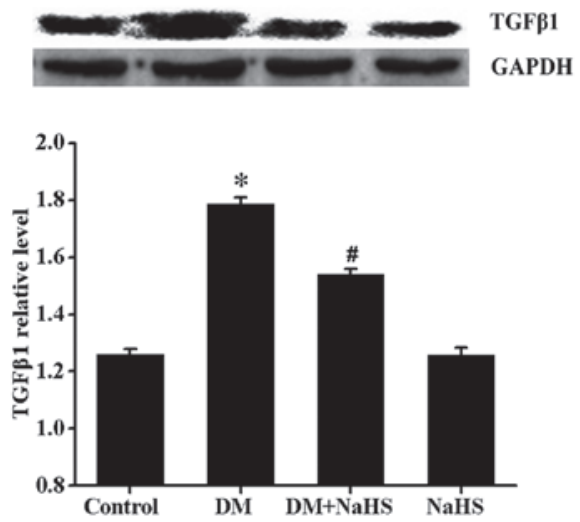

Figure 6. Expression of TGF $\beta 1$ in the myocardial tissues from each group. Data are expressed as the mean \pm standard deviation $(n=3)$. ${ }^{*} \mathrm{P}<0.05$ vs. the control group; ${ }^{\text {" }} \mathrm{P}<0.05$ vs. the $\mathrm{DM}$ group. TGF $\beta 1$, transforming growth factor $\beta 1$. was observed in the expression levels of PI3K and AKT1 between the control and NaHS groups.

\section{Discussion}

Prolonged hyperglycemia is hypothesized to induce metabolic disturbances and result in alterations to the balance of circulating hormones, which leads to the structural remodeling of the heart, including fibrosis (21). ECM is important in maintaining left ventricular geometry and ventricular function (22), and the suppression of ECM remodeling may be a therapeutic strategy to alleviate the progression of DCM. In the present study, rat models of diabetes were induced by intraperitoneal injection of STZ. In diabetic rats, symptoms of polyuria, polydipsia and polyphagia were observed, as well as blood glucose levels of $\geq 16.8 \mathrm{mM}$, indicating that the diabetic model 


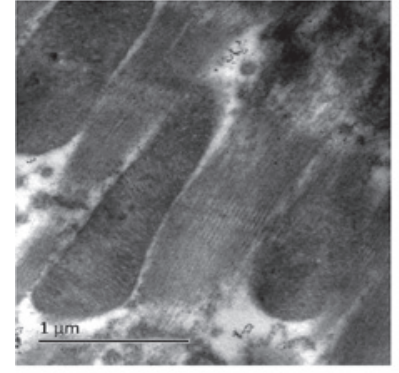

Control

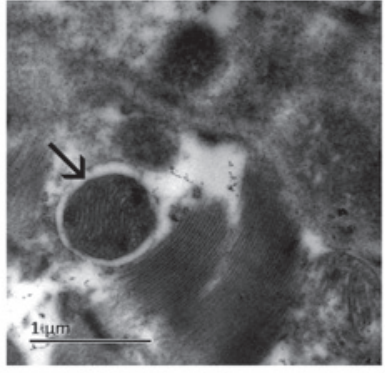

DM

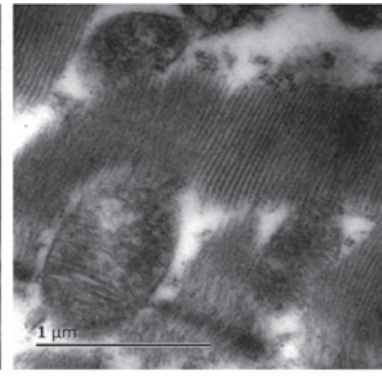

$\mathrm{DM}+\mathrm{NaHS}$

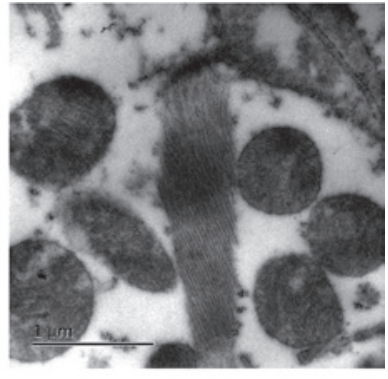

NaHS

Figure 7. Representative images of autophagosomes in rat myocardium samples from different groups detected by transmission electron microscopy. A representative autophagosome is indicated by a black arrow. DM, diabetic model; NaHS, sodium hydrosulfide.
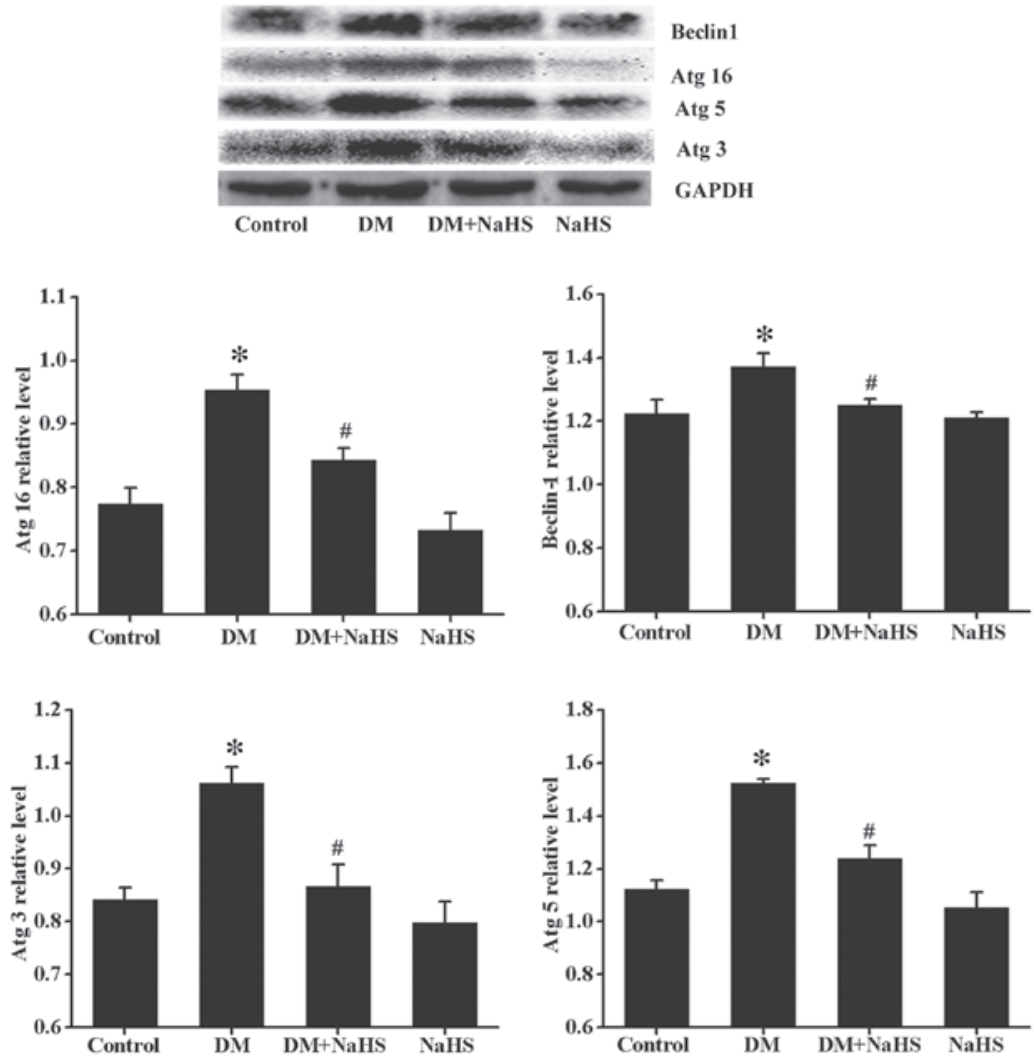

Figure 8. Expression of autophagy protein markers in the myocardial tissue samples from each group. Data are expressed as the mean \pm standard deviation $(\mathrm{n}=3)$. " $\mathrm{P}<0.05$ vs. the control group; ${ }^{\text {P }} \mathrm{P}<0.05$ vs. the DM group. DM, diabetes model; NaHS, sodium hydrosulfide; Atg, autophagy-related protein.
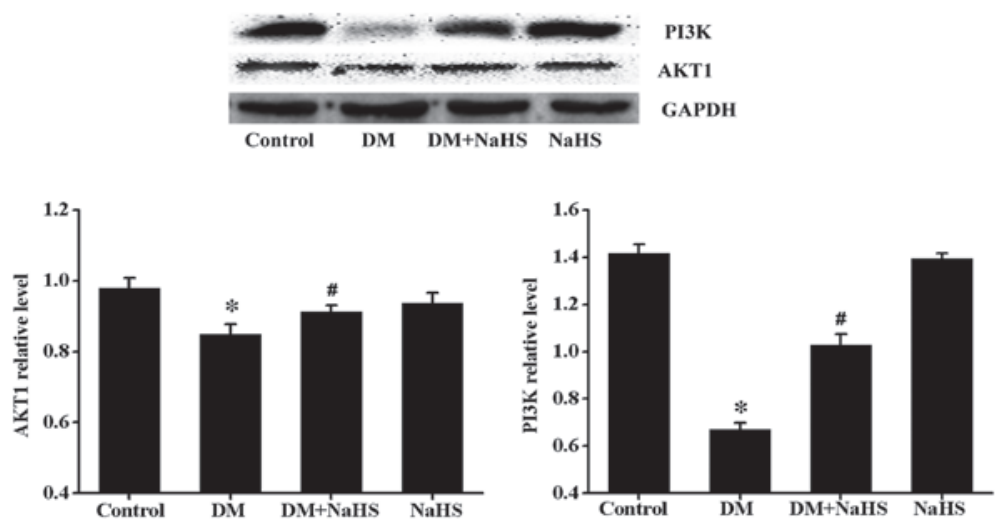

Figure 9. Expression of PI3K and AKT1 in the myocardial tissues from each group. Data are expressed as the mean \pm standard deviation $(\mathrm{n}=3)$. ${ }^{*} \mathrm{P}<0.05 \mathrm{vs.}$ the control group; ${ }^{"} \mathrm{P}<0.05$ vs the DM group. PI3K, phosphatidylinositol-4,5-bisphosphate 3-kinase; AKT1, RAC- $\alpha$ serine/threonine-protein kinase; DM, diabetic model; NaHS, sodium hydrosulfide. 
was successfully induced. At the end of the experiment, $\mathrm{H} \& \mathrm{E}$ and Masson staining were performed to evaluate the degree of fibrosis. The results demonstrated that diabetes increased myocyte cross-sectional area, induced a certain amount of disorganization of myocardial cell alignment and enhanced the deposition of collagen in the DM group. This suggests that myocardial damage was due to diabetes in the present study.

$\mathrm{H}_{2} \mathrm{~S}$ is an importantendogenous signaling molecule involved in the regulation of the cardiovascular system. Previous studies have demonstrated the cardioprotective role of $\mathrm{H}_{2} \mathrm{~S}$ in various models of cardiac injury, including ischemia/reperfusion injury, isoproterenol-induced heart failure and left ventricular remodeling induced by chronic alcohol consumption (23-25). Furthermore, in vitro research has demonstrated that $\mathrm{H}_{2} \mathrm{~S}$ protects against high-glucose-induced apoptosis in neonatal rat cardiomyocytes (26). Based on these findings, the present study hypothesized that administration of $\mathrm{NaHS}$ as $\mathrm{H}_{2} \mathrm{~S}$ donor would be of benefit in DCM. In addition, the current study demonstrated that the expression of CSE was significantly decreased in the DM group. CSE is the key enzyme that cleaves L-cysteine to release $\mathrm{H}_{2} \mathrm{~S}$ in heart, and downregulation of CSE results in decreased endogenous $\mathrm{H}_{2} \mathrm{~S}$ generation (6). It has been further suggested that NaHS treatment may alleviate myocardial damage induced by diabetes.

In the present study, previously described pathological abnormalities of the myocardium, which were induced by diabetes, were notably reversed following NaHS treatment, suggesting that $\mathrm{H}_{2} \mathrm{~S}$ ameliorates myocardial damage in rats with diabetes. To further investigate this result, the concentration of hydroxyproline and the expression of collagen I and collagen III were determined using the basic hydrolysis method and western blot analysis, respectively. Hydroxyproline content in the myocardium is used as a quantitative estimation of myocardial collagen production as it is widely understood that hydroxyproline constitutes $14 \%$ of the total amino acids of collagen $(27,28)$. It was observed that there were markedly higher levels of hydroxyproline and expression levels of collagen I and collagen III in the DM group; however, NaHS administration significantly downregulated the levels of hydroxyproline, collagen I and collagen III. These results also suggest that diabetes is associated with marked myocardial fibrosis and that $\mathrm{H}_{2} \mathrm{~S}$ exerts a protective effect on the normal myocardial structure.

MMPs and their endogenous inhibitors, TIMPs are the primary coordinators of collagen synthesis and degradation in cardiac tissue (29). MMPs are a family of enzymes that cleave matrix components and degrade fibrillar collagen, however, the products of degraded proteins serve as stimulators for collagen synthesis $(30,31)$. Previous studies have demonstrated that increased MMP expression levels and decreased expression levels of TIMP are accompanied by increased fibrosis, whereas downregulated MMP is associated with decreased deposition of collagen fibers $(32,33)$. TGF $\beta 1$ is widely accepted as a critical factor in fibrogenesis. It has been previously reported that TGF $\beta 1$ stimulates fibroblast proliferation and the production of ECM proteins, including fibronectin and collagen (34). Previous studies have also demonstrated that elevated expression levels of TGF $\beta 1$ are implicated in multiple fibrotic diseases, such as liver cirrhosis, pulmonary fibrosis and sclerosis (35-37). Consistent with the pathology results and altered expression of fibrosis markers mentioned above, NaHS treatment significantly alleviates diabetes-induced increases in the levels of MMPs and TGF $\beta 1$, and decrease of TIMP.

Myocardial fibrosis is commonly accompanied by excessive cardiomyocyte death $(38,39)$; an increased rate of cardiomyocyte death is characteristic of cardiac remodeling (40). Cardiac tissue consists of cardiomyocytes and extracellular matrix, excessive loss of myocardial cells increases the extracellular matrix and results in myocardial cells being replaced with fibrotic tissue. The accelerated death of cardiomyocytes promotes the progression of myocardial fibrosis.

Autophagy is the endogenous, tightly regulated cellular 'housekeeping' process responsible for the degradation of damaged and dysfunctional cellular organelles and protein aggregates (41). In a nutrient-deprived cell, autophagy is a cell-survival mechanism (42). However, prolonged activation of the autophagic signaling pathway results in cell death due to excessive self-digestion and degradation of essential constituents (43). For example, autophagy is involved in $\mathrm{T}$ cell death following the HIV-1 envelope protein binding to C-X-C chemokine receptor type 4 (44). In human leukemic cells, cell death resulting from downregulation of Bcl-2 was associated with increased autophagy (45). Furthermore, in the heart, autophagy is an essential form of cell death, along with apoptosis and necrosis (40). A previous study demonstrated that autophagy, rather than apoptosis, was the major model of cardiomyocyte death in the UM-X 7.1 hamster model of human dilated cardiomyopathy. Administration of granulocyte colony-stimulating factor treatment improved survival, ventricular function and remodeling, and reduced myocardial fibrosis; these beneficial effects were possibly associated with a reduction in autophagy (46). In the load-induced heart failure model, increased cardiac autophagy was observed. In addition, the inhibition of expression of Beclin-1, a protein required for early autophagosome formation, decreased cardiomyocyte autophagy and reduced pathological remodeling. By contrast, Beclin-1 overexpression increases autophagic activity and pathological remodeling (47). Another study regarding myocardial ischemia-reperfusion injury reported that although induction of autophagy during the ischemic phase was protective, further increases in autophagy during the reperfusion phase may induce cell death and appeared to be detrimental (48). These findings suggest that autophagy is closely associated with the occurrence of myocardial injury and the progression of heart failure. In the present study, diabetes triggered autophagy in the myocardium as evidenced by the increased number of autophagosomes and the increased expression of autophagy-associated proteins, the effects of which were ameliorated by NaHS treatment.

A central checkpoint that negatively regulates autophagy is mechanistic target of rapamycin, which is the downstream target of the PI3K/AKT signaling pathway. Upregulation of the PI3K/AKT1 signaling pathway has been demonstrated to suppress autophagy (49). In the present study, the PI3K/AKT1 signaling pathway was markedly inhibited in the myocardium of the DM group, whereas NaHS treatment was observed to activate PI3K/AKT1 in diabetic rats, which suggested that $\mathrm{H}_{2} \mathrm{~S}$ may protect against diabetes-induced cardiac autophagy via regulation of the PI3K/AKT1 signaling pathway. 
In conclusion, the results of the current study demonstrated that $\mathrm{H}_{2} \mathrm{~S}$ ameliorated myocardial fibrosis in rat diabetes models, and that its mechanism may involve inhibition of excessive activation of autophagy via regulation of PI3K/AKT1 pathway. The results provide a novel theoretical foundation for the antifibrosis mechanism of $\mathrm{H}_{2} \mathrm{~S}$ and suggests novel targets for DCM therapeutic strategies, although these findings require further verification. Further research should investigate whether 5' AMP-activated protein kinase, another important regulator of autophagy, is involved in diabetes and/or the $\mathrm{H}_{2} \mathrm{~S}$-induced cardiac autophagy response. In vitro studies using inhibitors to block autophagy or its regulatory pathway are required to further the present study.

\section{Acknowledgements}

The present study was supported by the National Natural Science Foundation of China (grant no. 81202830).

\section{References}

1. Pappachan JM,Varughese GI, Sriraman R and Arunagirinathan G: Diabetic cardiomyopathy: Pathophysiology, diagnostic evaluation and management. World J Diabetes 4: 177-189, 2013.

2. Asbun J and Villarreal FJ: The pathogenesis of myocardial fibrosis in the setting of diabetic cardiomyopathy. J Am Coll Cardiol 474: 693-700, 2006.

3. Falcão-Pires I and Leite-Moreira AF: Diabetic cardiomyopathy: Understanding the molecular and cellular basis to progress in diagnosis and treatment. Heart Fail Rev 17: 325-344, 2012.

4. Calvent JW, Coetzee WA and Lefer DJ: Novel insights into hydrogen sulfide-mediated cytoprotection. Antioxid Redox Sign 12: 1203-1217, 2010.

5. Yuan P, Xue H, Zhou L, Qu L, Li C, Wang Z, Ni J, Yu C, Yao T, Huang Y, et al: Rescue of mesangial cells from high glucose-induced over-proliferation and extracellular matrix secretion by hydrogen sulfide. Nephrol Dial Transplant 26: 2119-2126, 2011

6. Mani S, Li H, Untereiner A, Wu L, Yang G, Austin RC, Dickhout JG, Lhoták Š, Meng QH and Wang R: Decreased endogenous production of hydrogen sulfide accelerates atherosclerosis. Circulation 127: 2523-2534, 2013

7. King AL and Lefer DJ: Cytoprotective actions of hydrogen sulfide in ischaemia-reperfusion injury. Exp Physiol 96: 840-846, 2011.

8. Wang X, Wang Q, Guo W and Zhu YZ: Hydrogen sulfide attenuates cardiac dysfunction in a rat model of heart failure: A mechanism through cardiac mitochondrial protection. Biosci Rep 31: 87-98, 2011.

9. Zhao W, Zhang J, Lu Y and Wang R: The vasorelaxant effect of $\mathrm{H}_{(2)} \mathrm{S}$ as a novel endogenous gaseous K(ATP) channel opener. EMBO J 20: 6008-6016, 2001

10. Zhao W, Ndisang JF and Wang R: Modulation of endogenous production of $\mathrm{H}_{2} \mathrm{~S}$ in rat tissues. Can J Physiol Pharmacol 81: 848-853, 2003.

11. Kang B, Hong J, Xiao J, Zhu X, Ni X, Zhang Y, He B and Wang Z: Involvement of miR-1 in the protective effect of hydrogen sulfide against cardiomyocyte apoptosis induced by ischemia/reperfusion. Mol Biol Rep 41: 6845-6853, 2014.

12. Elrod JW, Calvert JW, Morrison J, Doeller JE, Kraus DW, Tao L, Jiao X, Scalia R, Kiss L, Szabo C, et al: Hydrogen sulfide attenuates myocardial ischemia-reperfusion injury by preservation of mitochondrial function. Proc Natl Acad Sci USA 104: 15560-15565, 2007.

13. Calvert JW, Elston M, Nicholson CK, Gundewar S, Jha S, Elrod JW, Ramachandran A and Lefer DJ: Genetic and pharmacologic hydrogen sulfide therapy attenuates ischemia-induced heart failure in mice. Circulation 122: 11-19, 2010.

14. Zhou X, Feng Y, Zhan ZB and Chen JC: Hydrogen sulfide alleviates diabetic nephropathy in a streptozotocin-induced diabetic rat model. J Biol Chem 289: 28827-28834, 2014.

15. Zhou X, An G and Chen J: Hydrogen sulfide improves left ventricular function in smoking rats via regulation of apoptosis and autophagy. Apoptosis 19: 998-1005, 2014.
16. Levine B and Yuan J: Autophagy in cell death: An innocent convict? J Clin Invest 115: 2679-2688, 2005.

17. Mizushima N, Levine B, Cuervo AM and Klionsky DJ: Autophagy fights disease through cellular self-digestion. Nature 451: 1069-1075, 2008.

18. Janku F, McConkey DJ, Hong DS and Kurzrock R: Autophagy as a target for anticancer therapy. Nat Rev Clin Oncol 8: 528-539, 2011.

19. YounceCW,WangKandKolattukudy PE: Hyperglycaemia-induced cardiomyocyte death is mediated via MCP-1 production and induction of a novel zinc-finger protein MCPIP. Cardiovasc Res 87: 665-674, 2010.

20. Zhu H, Rothermel BA and Hill JA: Autophagy in load-induced heart disease. Methods Enzymol 453: 343-363, 2009.

21. Wang L, Yuan T, Du G, Zhao Q, Ma L and Zhu J: The impact of 1,25-dihydroxyvitamin D3 on the expression of connective tissue growth factor and transforming growth factor- $\beta 1$ in the myocardium of rats with diabetes. Diabetes Res Clin Pract 104: 226-233, 2014.

22. Wang X, Lv H, Gu Y, Wang X, Cao H, Tang Y, Chen H and Huang C: Protective effect of lycopene on cardiac function and myocardial fibrosis after acute myocardial infarction in rats via the modulation of p38 and MMP-9. J Mol Histol 45: 113-120, 2014.

23. Andreadou I, Iliodromitis EK, Rassaf T, Schulz R, Papapetropoulos A and Ferdinandy P: The role of gasotransmitters $\mathrm{NO}, \mathrm{H}_{2} \mathrm{~S}$ and $\mathrm{CO}$ in myocardial ischaemia/reperfusion injury and cardioprotection by preconditioning, postconditioning and remote conditioning. Br J Pharmacol 172: 1587-1606, 2014.

24. Liu YH, Lu M, Xie ZZ, Hua F, Xie L, Gao JH, Koh YH and Bian JS: Hydrogen sulfide prevents heart failure development via inhibition of renin release from mast cells in isoproterenol-treated rats. Antioxid Redox Signal 20: 759-769, 2014.

25. Zhou X, Lu X, Xu W and Chen J: Protective effects of hydrogen sulfide against chronic alcohol intake-induced left ventricular remodeling in rats. Cardiovasc Drugs Ther 27: 221-227, 2013.

26. Zhou $X$ and Lu X: Hydrogen sulfide inhibits high-glucose-induced apoptosis in neonatal rat cardiomyocytes. Exp Biol Med (Maywood) 238: 370-374, 2013.

27. Xu X, Ding F, Pang J, Gao X, Xu RK, Hao W, Cao JM and Chen C: Chronic administration of hexarelin attenuates cardiac fibrosis in the spontaneously hypertensive rat. Am J Physiol Heart Circ Physiol 303: H703-H711, 2012.

28. Bish LT, Yarchoan M, Sleeper MM, Gazzara JA, Morine KJ, Acosta P, Barton ER and Sweeney HL: Chronic losartan administration reduces mortality and preserves cardiac but not skeletal muscle function in dystrophic mice. PLoS One 6: e20856, 2011.

29. Li YY, McTiernan CF and Feldman AM: Interplay of matrix metalloproteinases, tissue inhibitors of metalloproteinases and their regulators in cardiac matrix remodeling. Cardiovasc Res 46: 214-224, 2000.

30. Li YY, Feng YQ, Kadokami T, McTiernan CF, Draviam R, Watkins SC and Feldman AM: Myocardial extracellular matrix remodeling in transgenic mice overexpressing tumor necrosis factor alpha can be modulated by anti-tumor necrosis factor alpha therapy. Proc Natl Acad Sci USA 97: 12746-12751, 2000.

31. Maquart FX, Bellon G, Chaqour B, Wegrowski J, Patt LM, Trachy RE, Monboisse JC, Chastang F, Birembaut P and Gillery P: In vivo stimulation of connective tissue accumulation by the tripeptide-copper complex glycyl-L-histidyl-L-lysine-Cu2 $2^{+}$in rat experimental wounds. J Clin Invest 92: 2368-2376, 1993.

32. Dixon IM, Ju H, Reid NL, Scammell-La Fleur T, Werner JP and Jasmin G: Cardiac collagen remodeling in the cardiomyopathic Syrian hamster and the effect of losartan. J Mol Cell Cardiol 29: 1837-1850, 1997.

33. Cowan KN, Jones PL and Rabinovitch M: Regression of hypertrophied rat pulmonary arteries in organ culture is associated with suppression of proteolytic activity, inhibition of tenascin-C, and smooth muscle cell apoptosis. Circ Res 84: 1223-1233, 1999.

34. Spiekman M, Przybyt E, Plantinga JA, Gibbs S, van der Lei B and Harmsen MC: Adipose tissue-derived stromal cells inhibit TGF- $\beta 1$-induced differentiation of human dermal fibroblasts and keloid scar-derived fibroblasts in a paracrine fashion. Plast Reconstr Surg 134: 699-712, 2014.

35. Lee WR, Kim KH, An HJ, Kim JY, Lee SJ, Han SM, Pak SC and Park KK: Apamin inhibits hepatic fibrosis through suppression of transforming growth factor $\beta 1$-induced hepatocyte epithelial-mesenchymal transition. Biochem Biophys Res Commun 450: 195-201, 2014. 
36. Harris WT, Kelly DR, Zhou Y, Wang D, MacEwen M, Hagood JS Clancy JP, Ambalavanan N and Sorscher EJ: Myofibroblast differentiation and enhanced TGF-B signaling in cystic fibrosis lung disease. PLoS One 8: e70196, 2013.

37. Masola V,Zaza G, Secchi MF, Gambaro G, Lupo A and Onisto M: Heparanase is a key player in renal fibrosis by regulating TGF- $\beta$ expression and activity. Biochim Biophys Acta 1843: 2122-2128, 2014.

38. Radovits T, Korkmaz S, Mátyás C, Oláh A, Németh BT, Páli S, Hirschberg K, Zubarevich A, Gwanmesia PN, Li S, et al: An altered pattern of myocardial histopathological and molecular changes underlies the different characteristics of type- 1 and type-2 diabetic cardiac dysfunction. J Diabetes Res 2015: 728741, 2015.

39. Yu X, Zhang Q, Cui W, Zeng Z, Yang W, Zhang C, Zhao H Gao W, Wang X and Luo D: Low molecular weight fucoidan alleviates cardiac dysfunction in diabetic Goto-Kakizaki rats by reducing oxidative stress and cardiomyocyte apoptosis. J Diabetes Res 2014: 420929, 2014.

40. Nishida K and Otsu K: Cell death in heart failure. Circ J 72 . A17-21, 2008.

41. Dong Y, Undyala VV, Gottlieb RA, Mentzer RM Jr and Przyklenk K: Autophagy: Definition, molecular machinery, and potential role in myocardial ischemia-reperfusion injury. J Cardiovasc Pharmacol Ther 15: 220-230, 2010.

42. Dziedzic SA and Caplan AB: Autophagy proteins play cytoprotective and cytocidal roles in leucine starvation-induced cell death in Saccharomyces cerevisiae. Autophagy 8: 731-738, 2012.
43. Nishida K, Kyoi S, Yamaguchi O, Sadoshima J and Otsu K: The role of autophagy in the heart. Cell Death Differ 16: 31-38, 2009.

44. Espert L, Denizot M, Grimaldi M, Robert-Hebmann V, Gay B, Varbanov M, Codogno P and Biard-Piechaczyk M: Autophagy is involved in T cell death after binding of HIV-1 envelope proteins to CXCR4. J Clin Invest 116: 2161-2172, 2006.

45. Saeki K, Yuo A, Okuma E, Yazaki Y, Susin SA, Kroemer G and Takaku F: Bcl-2 down-regulation causes autophagy in a caspase-independent manner in human leukemic HL60 cells. Cell Death Differ 7: 1263-1269, 2000.

46. Miyata S, Takemura G, Kawase Y, Li Y, Okada H, Maruyama R, Ushikoshi H, Esaki M, Kanamori H, Li L, et al: Autophagic cardiomyocyte death in cardiomyopathic hamsters and its prevention by granulocyte colony-stimulating factor. Am J Pathol 168: 386-397, 2006.

47. Zhu H, Tannous P, Johnstone JL, Kong Y, Shelton JM, Richardson JA, Le V, Levine B, Rothermel BA and Hill JA: Cardiac autophagy is a maladaptive response to hemodynamic stress. J Clin Invest 117: 1782-1793, 2007.

48. Matsui Y, Kyoi S, Takagi H, Hsu CP, Hariharan N, Ago T, Vatner SF and Sadoshima J: Molecular mechanisms and physiological significance of autophagy during myocardial ischemia and reperfusion. Autophagy 4: 409-415, 2008.

49. Ma H, Guo R, Yu L, Zhang Y and Ren J: Aldehyde dehydrogenase 2 (ALDH2) rescues myocardial ischaemia/reperfusion injury: Role of autophagy paradox and toxic aldehyde. Eur Heart J 32: 1025-1038, 2011 